\title{
Cálculo dos Fatores de Intensidade de Tensão de uma Trinca Elíptica-hiperbólica Homofocal através do Método de Transformação Conforme ${ }^{1}$
}

G.F.P. SOARES ${ }^{2}$ C. WANG ${ }^{3}$, Departamento de Tecnologia, UNIJUÍ, 98700-000 Ijuí, RS, Brasil.

Resumo. Neste trabalho calculam-se os fatores de intensidade de tensão de uma trinca interna elíptica-hiperbólica homofocal. Tal problema foi investigado à luz dos conceitos da teoria da elasticidade e da mecânica da fratura através da análise complexa onde a transformação conforme (a trinca foi transformada em um ciclo unitário) e a integral de valor principal de Cauchy são envolvidas. Os resultados foram comparados com outros já obtidos numericamente. As fórmulas encontradas por este trabalho podem ser utilizadas como referências para determinar rapidamente os fatores de intensidade de tensão das trincas emitidas de uma elipse ou um ciclo.

\section{Introdução}

A existência de trincas em uma estrutura sempre é uma ameaça à sua segurança pois sua resistência diminui com a propagação destas trincas. Já a propagação ou não das trincas depende do campo de tensões ao redor de sua ponta, ou, ainda melhor, dos fatores de intensidade de tensões.

Nos problemas de propagação de trincas pode-se estudar três aspectos: se há ou não propagação e, no caso de haver, qual a direção e quantidade propagada. Todos envolvem diretamente os fatores de intensidade de tensões. Neste caso está sendo estudado o primeiro aspecto.

Os fatores de intensidade de tensões de muitas trincas originadas de geometria simples, como por exemplo, linhas retas, retangulares, circulares e elípticas com ramificações lineares conjugadas já foram estudadas numericamente [5]. No presente trabalho são calculados os fatores de intensidade de tensão de uma trinca elípticahiperbólica homofocal (Figura 1) pois a trincas deste tipo aparecem comumente em materiais geológicos como rochas e solos.

Este problema foi investigado analiticamente através da teoria da elasticidade, da mecânica da fratura [1] e da análise complexa envolvendo transformação conforme e a integral de valor principal de Cauchy.

\footnotetext{
${ }^{1}$ Trabalho de dissertação do mestrado em Modelagem Matemática da UNIJUÍ.

2 gilsonfps@bol.com.br

3 wang@unijui.tche.br; orientador
} 


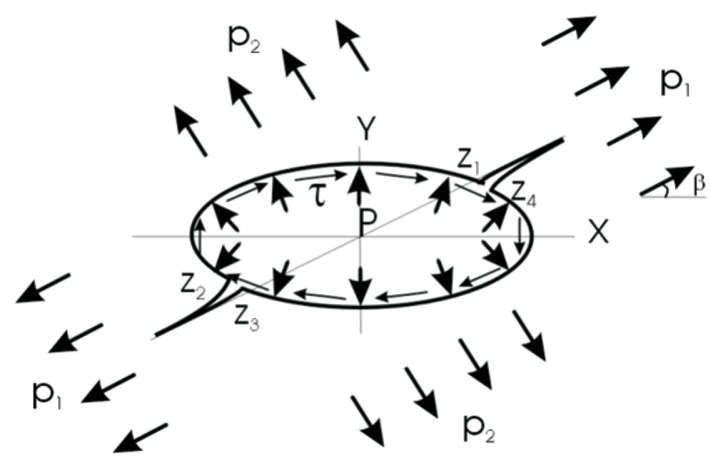

Figura 1: Trinca elíptica-hiperbólica homofocal sujeita à tensões.

\section{Apresentação do Problema}

A trinca em questão está sujeita a tensão normal $p$ e de cisalhamento $\tau$ no contorno da elipse e às tensões normais remotas $p_{1}$ e $p_{2}$,onde $p_{1}$ é direcionada pelo ângulo $\beta$ formado entre ela e o eixo $x$ positivo (Figura 1). A elípse simula um poro numa rocha subterrânea e a ramificação hiperbólica conjugada representa as trincas originadas da elípse. Os pontos $z_{1}, z_{2}, z_{3}$ e $z_{4}$ são os pontos de intersecção das trincas e o ponto $z_{0}$ é a extremidade da trinca. Na verdade $z_{1}$ e $z_{4}$ (e $z_{2}$ e $\left.z_{3}\right)$ são apenas os pontos dobrados de um mesmo ponto. Objetiva-se encontrar os fatores de intensidade de tensão em $z_{0}$, que determinam se a trinca propaga.

Muskhelishvili [2] expressa os componentes de tensões $\sigma_{x x}, \sigma_{y y}$ e $\sigma_{x y}$ em termos das funções analíticas complexas $\varphi_{1}(z)$ e $\psi_{1}(z)$

$$
\begin{gathered}
\sigma_{x x}+\sigma y y=2\left[\varphi_{1}^{\prime}(z)+\overline{\varphi_{1}^{\prime}(z)}\right] \\
\sigma_{y y}-\sigma_{x x}+2 i \sigma_{x y}=2\left[\bar{z} \varphi_{1}^{\prime \prime}(z)+\psi_{1}^{\prime}(z)\right] .
\end{gathered}
$$

A condição de contorno é

$$
f(\sigma)=\varphi_{1}(\sigma)+\overline{\sigma \varphi_{1}^{\prime}(\sigma)}+\psi_{1}(\sigma)
$$

onde $\sigma$ é o ponto acima do contorno. A integral

$$
f=i \int_{A}^{B}\left(X_{n}+i Y_{n}\right) d S+\text { const }=i(X+i Y)+\text { const }
$$

representa a resultante complexa $(\mathrm{X}, \mathrm{Y})$ das tensões do arco AB do contorno. Se este arco submete-se às tensões cisalhante $\tau$ e pressão $p$ (tensão normal), então, tem-se ainda de Timoshenko [6] que

$$
\begin{gathered}
X_{n} d S=[-\tau \cos (n, y)-p \cos (n, x)] d S=\tau d x-p d y, \\
i Y_{n} d S=i[\tau \cos (n, x)-p \cos (n, y)] d S=i(\tau d y+p d x),
\end{gathered}
$$




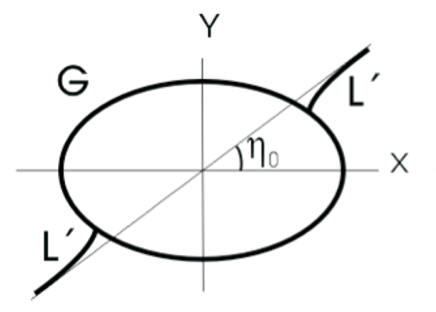

Plano z

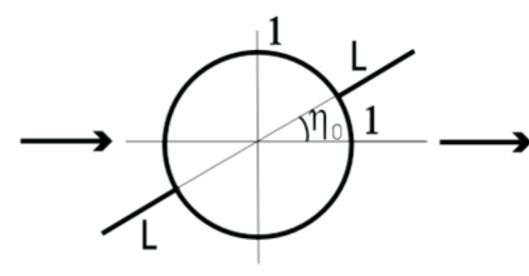

Plano $z_{1}$

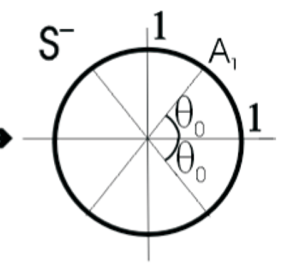

Plano $\zeta$

Figura 2: Transformação conforme $z \longrightarrow w(\zeta)$.

sendo $\cos (n, x)=\frac{d y}{d S}$ e $\cos (n, y)=-\frac{d x}{d S}$. Assim,

$$
f=i \int_{A}^{B}(\tau+i p) d z+\text { const }=i(\tau+i p)\left(z_{B}-z_{A}\right)+\text { const }
$$

$\mathrm{ou}$

$$
f=i N\left(z_{B}-z_{A}\right)+\text { const }
$$

onde $N=\tau+i p$. Se não há nenhum tipo de tensão no contorno, ou as tensões se equilibram estaticamente no contorno, então

$$
X=Y=0
$$

\section{Transformação Conforme}

Para facilitar a obtenção da solução do problema definida no domínio $(z)$ que tem um contorno complicado, é vantajoso transformar o domínio físico $z$ para outro domínio (notando por $\zeta$ ) que tem seu contorno relativamente simples. Denotamos esta transformação por

$$
z=w(\zeta)
$$

A Figura 2 mostra a transformação do contorno complicado do problema físico para um ciclo unitário. A transformação matemática é

$$
z=w(\zeta)=\frac{\alpha}{4}\left(\zeta+\zeta^{-1}\right) A_{1} \pm A_{2} \sqrt{\frac{\alpha^{2}}{4^{2}}\left(\zeta+\zeta^{-1}\right)^{2}-1}
$$

onde

$$
\begin{aligned}
& A_{1}=a \cos \eta_{0}+i b \sin \eta_{0}, \\
& A_{2}=b \cos \eta_{0}+i a \sin \eta_{0},
\end{aligned}
$$

sendo $\zeta=\rho e^{i \theta} \in S^{-}, a$ e $b$ os comprimentos dos meios eixos da elípse, $\eta_{0}$ o ângulo aproximativo da ramificação hiperbólica, $\alpha$ e $\theta_{0}$ os parâmetros que envolvem os comprimentos da hipérbole e $\cos \theta_{0}=\frac{2}{\alpha}$ (Figura 3). Essa transformação é conforme. De Timoshenko admite-se que (3.1) pode ser escrita como uma expressão de Laurent. 


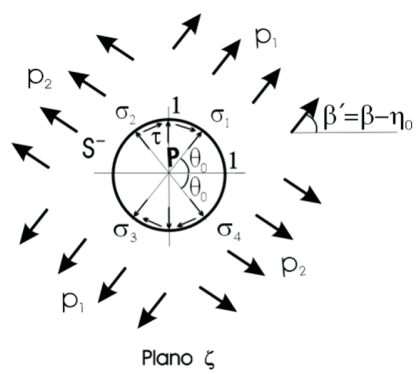

Figura 3: Plano $\zeta$.

Portanto,

$$
z=R \zeta+C_{0}+\frac{C_{1}}{\zeta}+\frac{C_{2}}{\zeta^{2}}+\cdots=R \zeta+w_{0}(\zeta)
$$

onde $R=\frac{\alpha}{4}\left(A_{1}+A_{2}\right)$ e $w_{0}(\zeta)$ é analítica em $S^{-}$.

\section{Função de Tensões}

De [2] sabe-se que as funções analíticas do campo de tensões são expressas por

$$
\begin{gathered}
\varphi_{1}(z)=\varphi(\zeta)=\Gamma R \zeta-\frac{X+i Y}{2 \pi(k+1)} \ln \zeta+\varphi_{0}(\zeta), \\
\psi_{1}(z)=\psi(\zeta)=\Gamma^{\prime} R \zeta+\frac{k(X-i Y)}{2 \pi(1+k)} \ln \zeta+\psi_{0}(\zeta) .
\end{gathered}
$$

Aplicando a condição de contorno (2.2), tem-se

$$
\begin{aligned}
& \varphi_{1}(z)=\varphi(\zeta)=\Gamma R \zeta+\varphi_{0}(\zeta), \\
& \psi_{1}(z)=\psi(\zeta)=\Gamma^{\prime} R \zeta+\psi_{0}(\zeta),
\end{aligned}
$$

com as funções analíticas $\varphi_{0}(\zeta) \in S^{-}$e $\psi_{0}(\zeta) \in S^{-}$representadas em séries da forma

$$
\begin{aligned}
& \varphi_{0}(\zeta)=\sum_{n=1}^{\infty} a_{n} \zeta^{-n}, \\
& \psi_{0}(\zeta)=\sum_{n=1}^{\infty} b_{n} \zeta^{-n},
\end{aligned}
$$

com $\varphi_{0}(\infty)=\psi_{0}(\infty)=0$ e as constantes complexas $\Gamma=\frac{1}{4}\left(p_{1}+p_{2}\right)$ e $\Gamma^{\prime}=$ $-\frac{1}{2}\left(p_{1}-p_{2}\right) e^{-2 i \beta^{\prime}}$. Ainda de Muskhelishvili, tem-se as condições de contorno

$$
\varphi(\sigma)+\frac{w(\sigma)}{\overline{w^{\prime}(\sigma)}} \overline{\varphi^{\prime}(\sigma)}+\overline{\psi(\sigma)}=f
$$




$$
\overline{\varphi(\sigma)}+\frac{\overline{w(\sigma)}}{w^{\prime}(\sigma)} \varphi^{\prime}(\sigma)+\psi(\sigma)=\bar{f}
$$

onde $\sigma=e^{i \theta} \in \gamma$ (extremidade da trinca no ciclo unitário). Multiplicando (4.4) e (4.5) por $\frac{1}{2 \pi i(\sigma-\zeta)}$ e integrando no contorno $\gamma$, encontra-se

$$
\begin{aligned}
& \frac{1}{2 \pi i} \int_{\gamma} \frac{\varphi(\sigma)}{\sigma-\zeta} d \sigma+\frac{1}{2 \pi i} \int_{\gamma} \frac{w(\sigma)}{\overline{w^{\prime}(\sigma)}} \frac{\overline{\varphi^{\prime}(\sigma)}}{\sigma-\zeta} d \sigma+\frac{1}{2 \pi i} \int_{\gamma} \frac{\overline{\psi(\sigma)}}{\sigma-\zeta} d \sigma=\frac{1}{2 \pi i} \int_{\gamma} \frac{f}{\sigma-\zeta} d \sigma \\
& \frac{1}{2 \pi i} \int_{\gamma} \frac{\overline{\varphi(\sigma)}}{\sigma-\zeta} d \sigma+\frac{1}{2 \pi i} \int_{\gamma} \frac{\overline{w(\sigma)}}{w^{\prime}(\sigma)} \frac{\varphi^{\prime}(\sigma)}{\sigma-\zeta} d \sigma+\frac{1}{2 \pi i} \int_{\gamma} \frac{\psi(\sigma)}{\sigma-\zeta} d \sigma=\frac{1}{2 \pi i} \int_{\gamma} \frac{\bar{f}}{\sigma-\zeta} d \sigma
\end{aligned}
$$

De $(3.1)$, temos

$$
\begin{gathered}
w^{\prime}(\zeta)=\frac{\alpha}{4}\left(1-\zeta^{-2}\right) \frac{A_{1} \sqrt{\frac{\alpha^{2}}{4^{2}}\left(\zeta+\zeta^{-1}\right)^{2}-1}+\frac{\alpha A_{2}}{4}\left(\zeta+\zeta^{-1}\right)}{\sqrt{\frac{\alpha^{2}}{4^{2}}\left(\zeta+\zeta^{-1}\right)^{2}-1}}, \\
\overline{w^{\prime}(\zeta)}=\frac{\alpha}{4}\left(1-\overline{\zeta^{-2}}\right) \frac{\overline{A_{1}} \sqrt{\frac{\alpha^{2}}{4^{2}}\left(\zeta+\zeta^{-1}\right)^{2}-1}+\frac{\alpha \overline{A_{2}}}{4}\left(\bar{\zeta}+\overline{\zeta^{-1}}\right)}{\sqrt{\frac{\alpha^{2}}{4^{2}}\left(\zeta+\zeta^{-1}\right)^{2}-1}} .
\end{gathered}
$$

Em $\sigma= \pm 1$ ocorre que (4.8) e (4.9) são anuladas. Desta forma, encontra-se a singularidade na equação (4.6). Se supormos que a função referente

$$
F(\omega)=\left[\frac{w(\omega)}{\overline{w^{\prime}(\omega)}} \overline{\varphi^{\prime}(\omega)}+\overline{\psi(\omega)}\right] \frac{\omega^{2}-1}{\omega^{2}-\zeta^{2}}
$$

satisfaz a condição de Hölder[2] para $\omega= \pm 1$, então, pela aplicação do conceito da integral de valor principal de Cauchy[2], pode-se alterar o caminho da integral para $c_{1}$, consistindo pelo caminho fechado: $c_{1-}, c_{1+}$ e o $\gamma$ menos os dois arcos pequenos em torno dos pontos $\sigma= \pm 1$, onde $c_{1-}$ e $c_{1+}$ são os meio ciclos centrados nos $\sigma=\mp 1$ respectivamente com o raio $r(r \longrightarrow 0)$ em $S^{+}$(Figura $4($ a)). Assim tem-se

$$
\begin{aligned}
& \frac{1}{2 \pi i} \int_{\gamma} \frac{\varphi(\sigma)}{\sigma-\zeta} d \sigma+\frac{1}{2 \pi i} \int_{c_{1}} \frac{w(\sigma)}{\overline{w^{\prime}(\sigma)}} \frac{\overline{\varphi^{\prime}(\sigma)}}{\sigma-\zeta} d \sigma+\frac{1}{2 \pi i} \int_{c_{1}} \frac{\overline{\psi(\sigma)}}{\sigma-\zeta} d \sigma \\
& -\lim _{\gamma \longrightarrow 0} \frac{1}{2 \pi i} \int_{c_{1 \pm}}\left[\frac{w(\sigma)}{\overline{w^{\prime}(\sigma)}} \overline{\varphi^{\prime}(\sigma)}+\overline{\psi(\sigma)}\right] \frac{d \sigma}{\sigma-\zeta}=\frac{1}{2 \pi i} \int_{\gamma} \frac{f}{\sigma-\zeta} d \sigma .
\end{aligned}
$$

Exceto a integral entre colchetes, as outras são integrais convencionais. Pode-se provar que a integral entre colchêtes é zero quando não há tensões nas superfícies perto da ponta da trinca [4]. Por isso simplifica-se (4.10) como segue:

$$
\frac{1}{2 \pi i} \int_{\gamma} \frac{\varphi(\sigma)}{\sigma-\zeta} d \sigma+\frac{1}{2 \pi i} \int_{c_{1}} \frac{w(\sigma)}{\overline{w^{\prime}(\sigma)}} \frac{\overline{\varphi^{\prime}(\sigma)}}{\sigma-\zeta} d \sigma+\frac{1}{2 \pi i} \int_{c_{1}} \frac{\overline{\psi(\sigma)}}{\sigma-\zeta} d \sigma=\frac{1}{2 \pi i} \int_{\gamma} \frac{f}{\sigma-\zeta} d \sigma .
$$




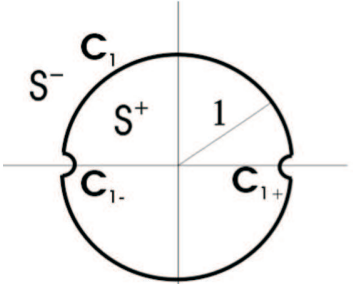

(a)

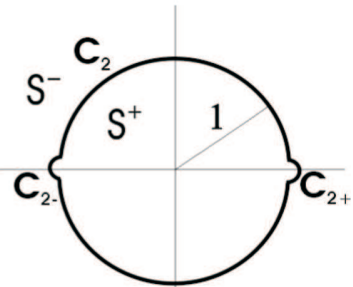

(b)

Figura 4: Plano $\zeta$.

Da mesma forma pode-se alterar o caminho da integral da equação (4.7) para $c_{2}$, consistindo no caminho fechado: $c_{2-}, c_{2+}$ e o $\gamma$ menos os dois arcos pequenos em torno dos pontos $\sigma= \pm 1$, onde $c_{2-}$ e $c_{2+}$ são os semi-ciclos centrados nos $\sigma=\mp 1$ respectivamente com raio $r(r \longrightarrow 0)$ em $S^{-}$(Figura 4(b)). Assim tem-se

$$
\frac{1}{2 \pi i} \int_{c_{2}} \frac{\overline{\varphi(\sigma)}}{\sigma-\zeta} d \sigma+\frac{1}{2 \pi i} \int_{c_{2}} \frac{\overline{w(\sigma)}}{w^{\prime}(\sigma)} \frac{\varphi^{\prime}(\sigma)}{\sigma-\zeta} d \sigma+\frac{1}{2 \pi i} \int_{\gamma} \frac{\psi(\sigma)}{\sigma-\zeta} d \sigma=\frac{1}{2 \pi i} \int_{\gamma} \frac{\bar{f}}{\sigma-\zeta} d \sigma .
$$

De acordo com (4.1) e (4.2), (4.11) e (4.12) podem ser escritas nas formas

$$
\begin{aligned}
& \frac{1}{2 \pi i} \int_{\gamma} \frac{\varphi_{0}(\sigma)}{\sigma-\zeta} d \sigma+\frac{1}{2 \pi i} \int_{c_{1}} \overline{\overline{w^{\prime}(\sigma)}} \frac{w(\sigma)}{\sigma-\zeta} \frac{\overline{\varphi_{0}^{\prime}(\sigma)}}{\sigma-\zeta} d \sigma+\frac{1}{2 \pi i} \int_{c_{1}} \frac{\overline{\psi_{0}(\sigma)}}{\sigma-\zeta} d \sigma \\
& =\frac{1}{2 \pi i} \int_{\gamma} \frac{f}{\sigma-\zeta} d \sigma-\frac{1}{2 \pi i} \int_{c_{1}} \frac{f_{0}}{\sigma-\zeta} d \sigma \\
& \frac{1}{2 \pi i} \int_{c_{2}} \frac{\overline{\varphi_{0}(\sigma)}}{\sigma-\zeta} d \sigma+\frac{1}{2 \pi i} \int_{c_{2}} \frac{\overline{w(\sigma)}}{w^{\prime}(\sigma)} \frac{\varphi_{0}^{\prime}(\sigma)}{\sigma-\zeta} d \sigma+\frac{1}{2 \pi i} \int_{\gamma} \frac{\psi_{0}(\sigma)}{\sigma-\zeta} d \sigma \\
& =\frac{1}{2 \pi i} \int_{\gamma} \frac{\bar{f}}{\sigma-\zeta} d \sigma-\frac{1}{2 \pi i} \int_{c_{2}} \frac{\overline{f_{0}}}{\sigma-\zeta} d \sigma
\end{aligned}
$$

$\operatorname{com} f_{0}=\Gamma R \sigma+\overline{\Gamma R} \frac{w(\sigma)}{\overline{w^{\prime}(\sigma)}}+\overline{\Gamma^{\prime} R \sigma}$.

De (4.3) sabe-se que $\varphi_{0}(\zeta)$ é analítica fora do $\gamma$ e $\varphi_{0}(\infty)=0$. Então, tem-se, segundo as fórmulas da integral de Cauchy,

$$
\frac{1}{2 \pi i} \int_{\gamma} \frac{\varphi_{0}(\sigma)}{\sigma-\zeta} d \sigma=\varphi_{0}(\zeta)-\varphi_{0}(\infty)=\varphi_{0}(\zeta)
$$

Sabe-se ainda que $\frac{w(\sigma)}{\overline{w^{\prime}(\sigma)}} \overline{\varphi_{0}^{\prime}(\sigma)}$ é o valor de contorno da função analítica $\frac{w(\zeta)}{\overline{w^{\prime}(\zeta)}} \overline{\varphi_{0}^{\prime}\left(\frac{1}{\zeta}\right)}$ em $S^{+}$. Portanto, de Cauchy obtém-se

$$
\frac{1}{2 \pi i} \int_{c_{1}} \frac{w(\sigma)}{\overline{w^{\prime}(\sigma)}} \frac{\overline{\varphi_{0}^{\prime}(\sigma)}}{\sigma-\zeta} d \sigma=0
$$




$$
\frac{1}{2 \pi i} \int_{c_{1}} \frac{\overline{\psi_{0}(\sigma)}}{\sigma-\zeta} d \sigma=0
$$

Assim (4.13) pode ser escrita da maneira

$$
\varphi_{0}(\zeta)=\frac{1}{2 \pi i} I_{1}-\Gamma R I_{2}-\overline{\Gamma^{\prime} R I_{3}}-\overline{\Gamma R I_{4}}
$$

onde

$$
\begin{gathered}
I_{1}=\int_{\gamma} \frac{f}{\sigma-\zeta} d \sigma, I_{2}=\frac{1}{2 \pi i} \int_{c_{1}} \frac{\sigma}{\sigma-\zeta} d \sigma \\
I_{3}=\frac{1}{2 \pi i} \int_{c_{1}} \frac{1}{\sigma(\sigma-\zeta)} d \sigma, I_{4}=\frac{1}{2 \pi i} \int_{c_{1}} \frac{w(\sigma)}{\overline{w^{\prime}(\sigma)}} \frac{d \sigma}{\sigma-\zeta}, \operatorname{com} \zeta \in S^{-} .
\end{gathered}
$$

Da equação (4.15) determina-se $\varphi_{0}(\zeta)$ que, levado até (4.14), permite o cálculo de $\psi_{0}(\zeta)$ através das fórmulas de Cauchy. Em conseqüência, pode-se obter o campo de tensões e do deslocamento. No entanto, o objetivo está no cálculo dos fatores de intensidade de tensão e não nas funções ora relatadas.

Assim, novamente das fórmulas de Cauchy, obtém-se

$$
\begin{gathered}
I_{2}=0, I_{3}=\frac{1}{\zeta} \Rightarrow \lim _{\zeta \longrightarrow 1} \frac{d I_{3}}{d \zeta}=-1, \\
I_{4}=\frac{A_{1}+A_{2}}{\overline{A_{1}+A_{2}}} \frac{1}{\zeta} \Rightarrow \lim _{\zeta \longrightarrow 1} \frac{d I_{4}}{d \zeta}=-\frac{A_{1}+A_{2}}{\overline{A_{1}+A_{2}}} .
\end{gathered}
$$

Para o cálculo de $I_{1}$ deve-se considerar o carregamento no contorno da elípse (Figura 4). Considerando (2.1) e sub-dividindo $f$ de forma que $f=f_{1}+f_{2}+f_{3}+f_{4}$ para os respectivos trechos tem-se

$$
\begin{gathered}
f_{1}=i N \int_{z_{4}}^{z} d z+i N z_{4}=i N z, \quad z \in \text { trecho } z_{4} z_{3}, \\
f_{2}=i N \int_{z_{4}}^{z_{3}} d z+i N z_{4}=i N z_{3}=i N z_{2}, \quad z \in \text { trecho } z_{3} z_{2}, \\
f_{3}=i N z, \quad z \in \text { trecho } z_{2} z_{1}, \\
f_{4}=i N z_{1}, \quad z \in \text { trecho } z_{4} z_{1},
\end{gathered}
$$

em que $z_{1}=z_{4}$ e $z_{2}=z_{3}$. Desta forma,

$$
I_{1}=\int_{\sigma_{4}}^{\sigma_{3}} \frac{f_{1}}{\sigma-\zeta} d \sigma+\int_{\sigma_{3}}^{\sigma_{2}} \frac{f_{2}}{\sigma-\zeta} d \sigma+\int_{\sigma_{2}}^{\sigma_{1}} \frac{f_{3}}{\sigma-\zeta} d \sigma+\int_{\sigma_{1}}^{\sigma_{4}} \frac{f_{4}}{\sigma-\zeta} d \sigma
$$

ou

$$
I_{1}=-i N\left[\int_{\sigma_{1}}^{\sigma_{2}} \frac{w(\sigma)}{\sigma-\zeta} d \sigma+\int_{-\sigma_{1}}^{-\sigma_{2}} \frac{w(\sigma)}{\sigma-\zeta} d \sigma+z_{2} \int_{\sigma_{2}}^{-\sigma_{1}} \frac{d \sigma}{\sigma-\zeta}+z_{1} \int_{-\sigma_{2}}^{\sigma_{1}} \frac{d \sigma}{\sigma-\zeta}\right]
$$

para $\sigma_{2}=-\sigma_{4}, \sigma_{1}=-\sigma_{3}$, e $z=w(\sigma)$. 
Resolvendo (4.16) chega-se em $\lim _{\zeta \longrightarrow 1} \frac{d I_{1}}{d \zeta}=\frac{\alpha A_{1} L^{\prime}}{2}\left(\pi-2 \theta_{0}-2 \cot \theta_{0}\right)+2 z_{1} i \csc \theta_{0}$. Assim, (4.13) assume a forma

$$
\begin{aligned}
\lim _{\zeta \longrightarrow 1} \frac{d}{d \zeta} \varphi_{0}(\zeta)= & -\frac{N}{2 \pi}\left[\frac{A_{1} i}{k}\left(\pi-2 \theta_{0}-2 \cot \theta_{0}\right)+2 z_{1} i \csc \theta_{0}\right] \\
& +\overline{\Gamma^{\prime} R}+\overline{\Gamma R} \frac{A_{1}+A_{2}}{\overline{A_{1}+A_{2}}}
\end{aligned}
$$

\section{Fatores de Intensidade de Tensão}

De Newman [3] tem-se que os fatores de intensidade de tensão dos modos I e II são determinados por

$$
K_{I}-i K_{I I}=\lim _{\zeta \longrightarrow 1} \sqrt{w(\zeta)-w(1)} \frac{\varphi^{\prime}(\zeta)}{w^{\prime}(\zeta)} 2 \sqrt{2 \pi}
$$

Mas, de (4.1), $\varphi^{\prime}(\zeta)=\Gamma R+\varphi_{0}^{\prime}(\zeta)$, então (5.1) fica

$$
K_{I}-i K_{I I}=\lim _{\zeta \longrightarrow 1} 2 \sqrt{2 \pi} \frac{\sqrt{w(\zeta)-w(1)}}{w^{\prime}(\zeta)}\left[\Gamma R+\varphi_{0}^{\prime}(\zeta] .\right.
$$

Calculando, temos

$$
\lim _{\zeta \longrightarrow 1} \frac{\sqrt{w(\zeta)-w(1)}}{w^{\prime}(\zeta)}=\sqrt{\frac{k \sqrt{1-k^{2}}}{2\left(A_{1} \sqrt{1-k^{2}}+A_{2}\right)}} .
$$

Levando (4.17) em (5.2) tem-se, para $\tan \theta_{0}=\frac{\sqrt{1-k^{2}}}{k}$ e $z_{1}=x_{1}+i y_{1}$,

$$
K_{I}-i K_{I I}=2 \sqrt{\frac{\pi \sqrt{1-k^{2}}}{k r_{0}}}\left[\left(B_{3} \cos \lambda_{0}+C_{3} \sin \lambda_{0}\right)-i\left(C_{3} \cos \lambda_{0}-B_{3} \sin \lambda_{0}\right)\right] .
$$

Assim, os fatores de intensidade de tensão são obtidos por

$$
\begin{aligned}
K_{I} & =2 \sqrt{\frac{\pi}{r_{0}} \tan \theta_{0}}\left(B_{3} \cos \lambda_{0}+C_{3} \sin \lambda_{0}\right), \\
K_{I I} & =2 \sqrt{\frac{\pi}{r_{0}} \tan \theta_{0}}\left(C_{3} \cos \lambda_{0}-B_{3} \sin \lambda_{0}\right),
\end{aligned}
$$

onde $r_{0}=\sqrt{\left(a \sqrt{1-k^{2}}+b\right)^{2} \cos ^{2} \eta_{0}+\left(b \sqrt{1-k^{2}}+a\right)^{2} \sin ^{2} \eta_{0}}$,

$\lambda_{0}=-\frac{1}{2} \arctan \left(\frac{b \sqrt{1-k^{2}}+a}{a \sqrt{1-k^{2}}+b} \tan \eta_{0}\right)$,

$B_{1}=-b \sin \eta_{0}\left(\pi-2 \theta_{0}-2 \cot \theta_{0}\right)-2 y_{1} \cot \theta_{0}, \quad C_{1}=a \cos \eta_{0}\left(\pi-2 \theta_{0}-2 \cot \theta_{0}\right)+$ $+2 x_{1} \cot \theta_{0}$,

$B_{2}=\left(p_{1}+p_{2}\right) \cos \eta_{0}-\left(p_{1}-p_{2}\right) \cos \left(2 \beta-3 \eta_{0}\right), C_{2}=\left(p_{1}+p_{2}\right) \sin \eta_{0}-\left(p_{1}-\right.$ $\left.p_{2}\right) \sin \left(2 \beta-3 \eta_{0}\right)$,

$B_{3}=\frac{a+b}{4} B_{2}-\frac{1}{2 \pi}\left(\tau B_{1}-p C_{1}\right), \quad C_{3}=\frac{1}{2 \pi}\left(\tau C_{1}+p B_{1}\right)-\frac{a+b}{4} C_{2}$. 


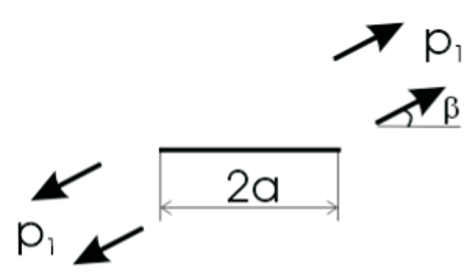

(a)

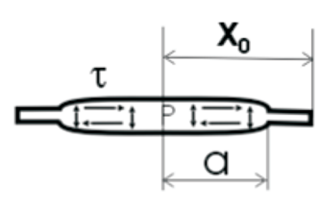

(b)

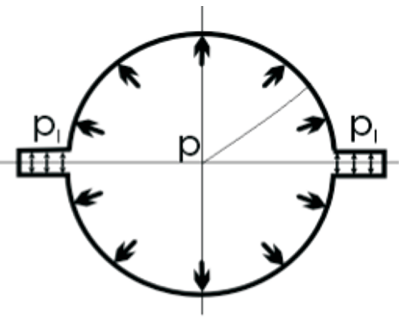

(c)

Figura 5: Trincas linear (a) e (b) e circular (c).

Se, na Figura 1, a trinca estiver sujeita às tensões normais $\left(p_{L}\right)$ e de cisalhamento $\left(\tau_{L}\right)$ nos contornos, então, pelo método da superposição, os parâmetros alteram-se para

$B_{3}=\frac{a+b}{4} B_{2}+\frac{1}{2 \pi}\left(\tau_{L} B_{4}+p_{L} C_{4}\right)-\frac{1}{2 \pi}\left[\left(\tau+\tau_{L}\right) B_{1}+\left(p_{L}-p\right) C_{1}\right]$,
$C_{3}=-\frac{a+b}{4} C_{2}+\frac{1}{2 \pi}\left(-\tau_{L} C_{4}+p_{L} B_{4}\right)+\frac{1}{2 \pi}\left[\left(\tau+\tau_{L}\right) C_{1}+\left(p-p_{L}\right) B_{1}\right]$,
$B_{4}=-\pi(a+b) \sin \eta_{0}, \quad C_{4}=\pi(a+b) \cos \eta_{0}$.

\section{Alguns Resultados}

\subsection{Trinca linear inclinada (Figura 5(a))}

Neste caso, para $b=\eta_{0}=p_{2}=p=\tau=0$, tem-se, para (5.3) e (5.4) respectivamente, essas fórmulas que já são muito conhecidas:

$$
K_{I}=p_{1} \sqrt{\pi a} \sin ^{2} \beta, \quad K_{I I}=p_{1} \sqrt{\pi a} \sin \beta \cos \beta .
$$

\subsection{Trinca linear sujeita a carregamento simétrico em partes das superfícies superior e inferior (Figura 5(b))}

Se $b=\eta_{0}=p_{1}=p_{2}=0$ então, de $(5.3)$ e (5.4), chega-se em

$$
K_{I}=\frac{2}{\pi} p \sqrt{\pi x_{0}} \sin ^{-1} \frac{a}{x_{0}}, \quad K_{I I}=\frac{2}{\pi} \tau \sqrt{\pi x_{0}} \sin ^{-1} \frac{a}{x_{0}},
$$

onde $\left(x_{0}, 0\right)$ são as coordenadas da extremidade da trinca. As fórmulas são idênticas às da literatura [5].

\subsection{Trinca originada de pressões internas no furo em um plano infinito (Figura 5(c))}

Se, agora, $\eta_{0}=p_{1}=p_{2}=\tau=\tau_{L}=0, p=p_{L}, a=b$, então (5.3) e (5.4) reduzem-se a

$$
K_{I}=2 p \sqrt{\pi} \sqrt{\frac{a \sqrt{1-k^{2}}}{k\left(\sqrt{1-k^{2}}+1\right)}}, \quad K_{I I}=0 .
$$


A Tabela 1 mostra alguns resultados para $K_{I}, \operatorname{com} a=1$.

Tabela 1: Valores de F em $K_{I}=F p \sqrt{\pi x_{0}}$.

\begin{tabular}{|c|c|c|c|c|c|}
\hline$\left(x_{0} \div a\right)$ & 1,01 & 1,50 & 2,00 & 2,50 & 4,00 \\
\hline$F($ Newman $)$ & 0.2188 & 0,9029 & 0,9670 & 0,9855 & 0,9976 \\
$F($ SoareseWang $)$ & 0,1979 & 0,8961 & 0,9682 & 0,9872 & 0,9980 \\
\hline
\end{tabular}

Abstract. This work calculated the stress intensity factors of hyperbolic cracks developed from the confocal ellipses. The two-dimensional holomorphic solutions were obtained on the base of elasticity theory, fracture mechanics and complex analysis where conformal mapping and Cauchy's principal integral are involved. The results are good in accordance to the numerical ones. The formulae can be used to determinate rapidly the stress intensity factors of cracks emanating from a circular or an ellipse hole in an infinite plate.

\section{Referências}

[1] T.L. Anderson, "Fracture Mechanics: Fundamentals and Applications", 2th ed., CRC Press, 1994.

[2] N.I. Muskhelishvili, "Some Basic Problems of the Mathematical Theory of Elasticy", Noordhoof International, Leyden, 1953.

[3] J.C. Newman, An improved method of collocation for the stress analysis of cracked plates with various shaped boundaries, NASA, TN-D6376 (1971).

[4] W.C. Qian e K.Y. Ye, "Mecânica da Elasticidade", Científica, Pequim, 1954.

[5] H. Tada, P.C. Paris e G.R. Irwin, "The Stress Analysis of Cracks Handbook", Del Research Corp., Hellertown, Pa, 1973.

[6] S.P. Timoshenko e J.N. Goodier, "Teoria da Elasticidade", Guanabara Dois, Rio de Janeiro, 1980. 